\title{
Estresse ocupacional na administração pública brasileira: quais os fatores impactantes?*
}

\author{
Moises Balassiano** \\ Elaine Tavares*** \\ Roberto da Costa Pimenta****
}

SumÁrio: 1. Introdução; 2. Aspectos teóricos; 3. Aspectos metodológicos; 4. A análise dos dados; 5 . Considerações finais.

Summary: 1. Introduction; 2. Theoretical aspects; 3. Methodological aspects; 4. Data analysis; 5. Final remarks.

Palavras-chave: estresse ocupacional; administração pública; modelo de equações estruturais.

KEY WORDs: occupational stress; public administration; structural equation model.

Neste artigo é examinado o estresse ocupacional no ambiente organizacional da administração pública brasileira. Na recensão teórica, são indicados os principais estressores organizacionais apontados na literatura especializada e identificados efeitos destes estressores sobre a saúde e o desempenho do indivíduo. A pesquisa empírica utilizou dados de uma investigação realizada com base em uma amostra de 242 servidores públicos federais. Na identificação dos estressores e dos sintomas de estresse, bem como a forma como eles se relacionam, foi utilizado um modelo

\footnotetext{
* Artigo recebido em mar. 2009 e aceito em maio 2010.

** PhD em psicologia quantitativa pela University of Illinois (EUA). Professor da Fucape Business School. Endereço: Av. Fernando Ferrari, 1358 - Boa Vista - CEP 29075-505, Vitória, ES, Brasil. E-mail: moises@fucape.br.

*** Doutora em administração pela Escola Brasileira de Administração Pública e de Empresas da Fundação Getulio Vargas (Ebape/FGV). Pós-doutoranda no Centre d'Etude et de Recherche en Gestion Aix Marseille, Université Aix-Marseille III - Aix-en-Provence — France. Professora da Ebape/FGV. Endereço: 3 Rue du Bras D’Or. BT D - 13100, Aix-en-Provence, France. E-mail: elaine.tavares@fgv.br.

$* * * *$ Doutor em administração pela Ebape/FGV. Pesquisador da Ebape/FGV. Endereço: Praia de Botafogo, 190, 5o andar - Botafogo - CEP 22250-900, Rio de Janeiro, RJ, Brasil. E-mail: roberto.pimenta@fgv.br.
} 
de equações estruturais (SEM). Com base nesse modelo, foram identificados três tipos de estressores ambientais: emoção, social e mobilidade, na forma percebida pelos servidores. Foram também identificados dois tipos de estresse: o psicológico e o fisiológico. Os resultados revelam que apenas o fator emocional presente no ambiente de trabalho nas organizações públicas influencia o estresse ocupacional psicológico, entre os fatores estudados. Espera-se que os resultados possam contribuir para orientar gestores públicos a criar um ambiente propício ao trabalho, com melhor qualidade de vida, gerando condições favorecedoras a altos padrões de produtividade na administração pública brasileira.

Occupational stress in the Brazilian public administration: what are the impacting factors?

In this article, we examine occupational stress in the organizational environment of the Brazilian public administration. In the theoretical review, the major organizational stressors highlighted in the literature are pointed out and their effects on health and performance of individuals are identified. The empirical research used data from a survey based on a sample of 242 federal public servants. A Structural Equation Model (SEM) was used to identify the stressors, the related stress symptoms, as well as the way they relate with each other. Three types of environmental stressors has been identified, namely: emotion, and social mobility, as perceived by the servants. The symptoms were classified according to two types of stress: psychological and physiological. The results show that only the emotional factor present in the workplace, in public organizations, influences the psychological occupational stress, among the factors studied. It is expected that the present results may help public managers to create better environment and a workplace favorable with high standards of productivity in the Brazilian public administration.

\section{Introdução}

O estresse no trabalho tem sido um tema amplamente discutido na literatura nos últimos anos. A natureza do estresse ocupacional, seus mecanismos e suas consequências para a saúde e o desempenho do trabalhador têm sido objeto de um número significativo de pesquisas (Cavanaugh, 2000; Cohen et al., 1995; Evans e Steptoe, 2001; Mackie, Holahan, e Gottlieb, 2001; Pollard, 2001; Wang e Patten, 2001).

O interesse acerca do tema se deve à preocupação dos pesquisadores com as várias consequências negativas que podem decorrer do estresse. Theorell (1985) demonstrou que, quando as condições de trabalho forem percebidas como estressantes de maneira permanente, poderão existir efeitos sobre o bem-estar psicológico e físico. Spielberger e Reheiser (1994) ressaltaram que 
o estresse no local de trabalho resulta em custos muito elevados para indivíduos, para organizações e para a sociedade.

Há um consenso na literatura de que o estresse intenso ou prolongado pode ter impacto negativo na saúde física e mental de uma pessoa (Cooper et al., 2001), podendo, em situações extremas, causar doenças cardíacas, dores generalizadas, distúrbios gastrointestinais, ansiedade e depressão (Johnson et al., 2005). A psicopatologia do trabalho coloca o sofrimento no centro da relação psíquica entre o homem e o trabalho. Dejours $(1992,1996)$ busca compreender como os trabalhadores, em sua maioria, conseguem preservar um equilíbrio psíquico e manter-se na normalidade, por meio de suas estratégias defensivas, em que a loucura seria o mais esperado, devido à exploração do sofrimento no ambiente organizacional.

Segundo Albrecht (1990), o estresse é uma doença que se tornou recorrente e está trazendo um elevado custo em termos de saúde e bem-estar emocional. O estado prolongado de preocupação, alerta e ansiedade, que caracteriza uma forte carga de estresse, se transformou num grande inimigo das pessoas (Albrecht, 1990; Moraes e Kilimnik, 1992).

O relaxamento na busca por um ambiente de trabalho propício para o bem-estar e para a produtividade, as doenças ocupacionais, mentais e físicas, refletem, em termos financeiros, o custo oculto do estresse no trabalho (Figueroa et al., 2001). Trabalhadores estressados tendem a uma diminuição no desempenho e na qualidade de seu trabalho e a gerar um aumento dos custos das organizações com problemas de saúde e com o aumento do absenteísmo, da rotatividade e do número de acidentes no local de trabalho (Meng-Jinn e Cunradi, 2008; Jex, 1998; Rees, 1995).

Nesta perspectiva, Santos e colaboradores (1999) consideram que pesquisar o estresse ocupacional constitui uma tentativa de conhecer melhor as variáveis que interferem no ajustamento do homem às mudanças das condições de trabalho, e o que pode ser feito para facilitar esta relação, promovendo melhoria da qualidade de vida do trabalhador e, assim, melhores condições para o incremento da produtividade.

Diversas pesquisas conceituam o estresse ocupacional de formas distintas (Rees e Redfern, 2000). Jex (1998), por exemplo, classifica as definições de acordo com três aspectos: (1) estímulos do ambiente de trabalho, conhecidos por estressores organizacionais, que exigem respostas adaptativas por parte do funcionário e que excedem sua habilidade de enfrentamento; (2) as diversas respostas que os indivíduos emitem quando expostos aos estressores; e (3) o processo geral em que demandas do trabalho têm impacto nos funcionários. De acordo com esta taxonomia, os estudos podem se basear nos 
estressores organizacionais, nas respostas do indivíduo a esses estressores ou nas diversas variáveis presentes no processo estressor-resposta.

No entanto, os principais estudos da área indicam que as percepções dos indivíduos são mediadoras do impacto do ambiente de trabalho sobre o indivíduo. É preponderante a ideia de que, para algo na organização ser um estressor, ele precisa ser percebido como tal pelo funcionário (Paschoal e Tamayo, 2004).

Desta ótica, o estresse ocupacional pode ser definido como um processo em que o indivíduo percebe demandas do trabalho como estressores, os quais, ao exceder sua habilidade de enfrentamento, provocam no sujeito reações negativas (Paschoal e Tamayo, 2004).

Esta definição está de acordo com o modelo de Michigan, apresentado por Seegers e Van Elderen. Segundo os autores:

O estresse é o resultante da percepção sobre a discordância entre as exigências da tarefa e os recursos pessoais para cumprir as ditas exigências. Uma pessoa pode sentir esta discordância como desafio e, em consequência, reagir dedicando-se à tarefa. Caso contrário, se a discordância é percebida como ameaçadora, então o trabalhador enfrentar-se-á com uma situação estressante negativa, que pode conduzi-lo a evitar a tarefa (Seegers e Van Elderen, 1996:213).

Neste sentido, qualquer intervenção em relação ao estresse ocupacional necessita identificar corretamente os estressores organizacionais. O sucesso de qualquer esforço para minimizar o estresse e maximizar a satisfação no trabalho depende deste diagnóstico, porque diferentes tipos de estresse requerem diversas ações (Fulcheri et al., 1995). Por esta razão, os estressores despertam tanto interesse (Steinmetz e Schmidt, 2010).

O estresse ocupacional está presente em qualquer profissão ou nível hierárquico (Albrecht, 1990; Mota et al., 2008, Laranjeira, 2009). A aptidão de criar e manter um ambiente com presença reduzida de estressores organizacionais é uma exigência crescente e todo administrador deve estar capacitado para gerir e reduzir o próprio estresse e para auxiliar na diminuição das tensões de seus subordinados. Além disto, é necessário admitir que haja estressores inerentes à natureza da atividade, à cultura organizacional e às interações humanas. Ainda assim, a identificação dos estressores é essencial para que se possa adequar o perfil dos cargos à convivência com estes estressores.

Devido à sua relevância para o entendimento do tema estresse ocupacional, os estressores ocupacionais têm sido pesquisados em muitas profissões, como professores (Travers e Cooper, 1993), profissionais de saúde (Cooper et 
al., 1999), enfermeiras e assistentes sociais (Kahn, 1993; Chen et al., 2009, Admi e Moshe-Eilon, 2010), policiais militares (Costa et al., 2007), militares (Campbell e Nobel, 2009) e gestores (Skakon et al., 2011).

No Brasil, essas investigações abrangem categorias distintas, como professores do ensino superior (Marques e Paiva, 1999; Ayres et al., 1999), magistrados (Lipp e Tanganelli, 2002), gestores de empresa pública (Couto e Paiva, 2008), bancários (Veloso e Pimenta, 2004), funcionários do setor automobilístico (Santos et al., 1999) e caixas de supermercado (Couto e Moraes, 1999).

No que diz respeito ao campo da administração pública, percebe-se que ainda há uma carência de pesquisas sobre o estresse ocupacional no Brasil. Neste contexto, uma pesquisa sobre o estresse ocupacional parece oportuna e relevante. Como consequência da significativa transformação do modelo de gestão das organizações públicas ocorrida nos últimos 10 anos, o ambiente dessas organizações incorporou uma nova dinâmica de trabalho que pressupõe novas habilidades e competências dos indivíduos.

Diante dessa nova perspectiva, inerente ao mundo digitalizado e que pressupõe a implementação de novas práticas gerenciais e a adoção de novas tecnologias, o ambiente de trabalho nas organizações públicas sofreu diversas alteraç̃̃es com o propósito básico de alcançar um desempenho mais produtivo.

Neste cenário, esta pesquisa tem o objetivo de identificar estressores próprios do âmbito do trabalho, no funcionalismo público no Brasil. Mais especificamente, desenvolveu-se a presente pesquisa para investigar a influência do ambiente do trabalho no estresse de servidores públicos federais.

A busca de evidências empíricas, que permitam inferir considerações relativas à percepção dos servidores públicos federais quanto à influência do ambiente de trabalho no desempenho de suas tarefas, ratifica a importância do estudo para o campo das organizações públicas e estimula o debate sobre o tema. Isto é especialmente oportuno em face do momento ímpar de profissionalização da função pública a partir da Reforma do Aparelho do Estado e das recentes inovações tecnológicas, que imprimiram mudanças substantivas no ambiente de trabalho da administração pública brasileira.

\section{Aspectos teóricos}

A pesquisa acadêmica utiliza uma grande diversidade de estressores organizacionais (Johnson et al., 2005). Estes estressores podem ser de natureza física (por exemplo, barulho, ventilação e iluminação do local de trabalho) ou psicossocial, e estes últimos têm despertado mais interesse nos psicólogos organizacionais. 
Ao investigarem os estressores organizacionais, Cooper e Marshall (1976) definiram cinco fontes de estresse:

(1) fatores intrínsecos ao trabalho, incluindo aspectos como baixas condições de trabalho, sobrecarga de informação, pressão de prazos, mudanças tecnológicas. A ambiência física presente em fatores como a qualidade do ar, a iluminação, a decoração, o barulho e o espaço pessoal pode influenciar o humor e o estado mental das pessoas;

(2) papel na organização, incluindo ambiguidade e conflito de papéis. A ambiguidade de papéis ocorre quando o indivíduo não tem uma visão clara sobre os objetivos de seu trabalho, da expectativa que seus colegas têm sobre ele e do escopo e responsabilidade de sua função;

(3) desenvolvimento de carreira, incluindo falta de segurança no emprego, falta ou excessos de promoções e obsolescência;

(4) relacionamentos no trabalho, incluindo relacionamentos com os colegas e com os superiores. Lidar com os chefes, pares e subordinados pode afetar bastante a forma com que o indivíduo se sente ao fim do dia; e

(5) estrutura e clima organizacional, incluindo baixo envolvimento na tomada de decisão e em questões políticas.

Entre os fatores intrínsecos ao trabalho, de acordo com Glowinkowski e Cooper (1987), a sobrecarga de trabalho tem recebido considerável atenção dos pesquisadores. Este estressor pode ser dividido em dois níveis: quantitativo e qualitativo. A sobrecarga quantitativa diz respeito ao número excessivo de tarefas a serem realizadas; isto é, a quantidade de tarefas encontra-se além da disponibilidade do trabalhador. A sobrecarga qualitativa refere-se à dificuldade do trabalho, ou seja, o indivíduo depara-se com demandas que estão além de suas habilidades ou aptidões (Glowinkowski e Cooper, 1987; Jex, 1998).

Nos relacionamentos no ambiente de trabalho, a maioria das ocupações envolve interações entre pessoas, seja entre colegas de mesmo nível hierárquico, superiores e subordinados ou entre funcionários e clientes, que podem gerar outra fonte de estresse (Glowinkowski e Cooper, 1987; Jex, 1998). Os estudos de Iwata e Suzuki (1997) e de Ramirez e colaboradores (2008) observaram que o suporte social se relacionou negativamente ao estresse.

Em relação à estrutura e ao clima organizacional, outro tipo de estressor, comumente relatado na literatura, refere-se ao controle/autonomia no trabalho (Johnson et al., 2005). O controle tem sido tratado em termos da 
autonomia do trabalhador em relação às decisões e aos métodos de trabalho (Paschoal e Tamayo, 2004).

Há ainda variáveis pessoais que influenciam o estresse ocupacional. O estilo de enfrentamento do funcionário diante dos eventos estressores consiste na principal variável individual, representando, ainda, um ponto central de muitos estudos (Iwata e Suzuki, 1997).

De forma mais abrangente, Cooper e Baglioni (1988) propuseram que o estresse é resultado da interação de várias fontes de pressão com o indivíduo. Com relação a essas fontes de pressão no trabalho, Cooper e colaboradores $(1988,1999)$ identificaram como estressores: (i) fatores intrínsecos ao trabalho, (ii) os relacionamentos interpessoais, (iii) a satisfação do trabalhador em termos de carreira e perspectivas futuras; e (iv) o clima e a estrutura organizacionais.

Além das fontes de pressão, Cooper e colaboradores $(1988,1999)$ também identificaram que o estresse ocupacional tem sintomas físicos e mentais, que podem se mostrar, no nível individual, por dores, depressão, alienação, apatia e ansiedade.

Dejours (1992, 1996), ao estudar o prazer e o sofrimento ocasionados pelo trabalho, evidenciou que a exploração do sofrimento não cria doenças mentais específicas, pois as pressões no ambiente organizacional acabam retirando das organizações os sujeitos que sofrem de sintomas mentais. As pesquisas relativas à teoria do estresse estão mais posicionadas no domínio no qual o psiquismo dos trabalhadores não sai da normalidade (Dejours, 1996).

Estas pesquisas apontam, entretanto, uma alternativa ao adoecer psíquico, que reside na somatização das doenças (Dejours, 1992:126). A competição e o conflito nas organizações têm efeitos relacionados à saúde dos executivos. Os efeitos, a longo prazo, são debilitantes e expõem os executivos a um grande número de riscos médicos e psicológicos à saúde (Mota et al., 2008).

Tal como os resultados de Cooper, Elkins e colaboradores (2010) também mostraram que o estresse ocupacional está associado a sintomas de depressão e ansiedade, como distúrbios de sono, dificuldade de manter atenção e fastio. Efeitos similares foram apontados pela Universidade de Loughborough (2009), que atribuiu ao estresse ocupacional dores, enjoos, ansiedade, dificuldades para dormir e falta de concentração. A estafa é um risco para o executivo e, além dos problemas psicológicos, apresenta também sintomas físicos, tais como dores, insônia e falta de fôlego (Quick et al., 2003).

Especificamente na área da administração pública, o departamento de saúde e segurança da Federação dos Funcionários Públicos do Estado de Nova York (New York State Public Employees Federation, 2010) identificou 
como efeitos do estresse ocupacional problemas de estômago, distúrbios de sono e fadiga.

\section{Aspectos metodológicos}

Consoante o objetivo precípuo do trabalho, qual seja, identificar e relacionar os estressores provenientes do ambiente do trabalho, foi delineado um estudo exploratório que permitisse avaliar a dinâmica da estrutura das relações das associações envolvendo um elenco de possíveis estressores e sintomas predefinidos. Os princípios metodológicos que nortearam a condução da investigação são, a seguir, apresentados.

\section{Amostra}

Visando a obtenção dos dados que permitissem a identificação dos estressores e sintomas do estresse ocupacional, foi selecionada uma amostra constituída por 242 funcionários públicos federais civis ativos filiados ao Sindicato dos Trabalhadores do Serviço Público Federal no Estado do Rio de Janeiro (Sintrasef). Esta entidade agrega servidores de aproximadamente 70 órgãos públicos federais, da administração direta e indireta, no estado do Rio de Janeiro.

O perfil da amostra foi marcado, preponderantemente, por servidores do sexo masculino (69,5\%), com faixa etária acima de 31 anos (90,5\%). Esse perfil foi comparado com os dados disponíveis sobre a população, em que pode ser observada a similaridade desejada. Segundo o Boletim Estatístico de Pessoal, emitido pelo Ministério do Planejamento, Orçamento e Gestão do governo brasileiro (Brasil, 2010), o estado do Rio de Janeiro possui 103.479 servidores públicos federais civis ativos. Destes, 50,8\% são do sexo masculino, com idade média de 47 anos.

\section{Os dados}

O instrumento de coleta dos dados foi um questionário constituído de duas seções, com itens consolidados a partir da revisão bibliográfica. Na primeira seção, foram apresentadas questões fechadas, de acordo com uma escala de diferencial semântico de cinco pontos que tinham como objetivo descrever o ambiente de trabalho. Na segunda, foram apresentados itens binários, na 
forma de check-list, que indagavam a possível presença, ou não, de sintomas relacionados ao estresse, tal como percebida pelos respondentes.

Os questionários foram aplicados no próprio local de trabalho dos servidores, após breve explicação do objetivo do estudo e do conteúdo das questões levantadas.

\section{Os construtos e seus indicadores}

Com o propósito de se identificar quantas e quais dimensões poderiam ser definidas a partir das variáveis de meio ambiente e as de sintomas de estresse, submeteram-se, primeiramente, os dados a uma análise fatorial exploratória. As relações entre as variáveis observáveis definiram uma estrutura latente para o meio ambiente e outra para os sintomas de estresse. Em ambos os casos, as variáveis que não carregaram de modo significativo em qualquer fator, ou cujo grupamento deixou de fazer algum sentido teórico ou prático, foram alijadas do estudo. Contribuiu para a obtenção de um resultado mais confiável o estabelecimento do critério de maximização do coeficiente alfa de Cronbach (1951), visando aumentar a fidedignidade do construto por meio de maior consistência interna de seus indicadores.

A avaliação da adequação do método utilizado no estudo à base de dados foi feita a partir de dois indicadores descritos no quadro 1: o teste de Kaiser-Meyer-Oklin para a adequação do tamanho da amostra e o teste da esfericidade da matriz das correlações entre as variáveis. No primeiro caso, o valor da estatística, 0,783, mostrou-se meritório para a amostra de 250 servidores, enquanto o teste da esfericidade rejeitou a hipótese nula de que a matriz das associações entre as variáveis pudesse ser considerada diagonal, caso que tornaria o método de análise adotado inadequado.

\section{Quadro 1}

\section{Teste KMO e da esfericidade de Bartlett}

\begin{tabular}{|l|l|r|}
\hline \multicolumn{2}{|c|}{ KAISER-MEYER-OKLIN MEASURE OF SAMPLING ADEQUACY. } & 0,783 \\
\hline BARTLETT'S TEST OF SPHERICITY & Approx. Chi-Square & 1945,792 \\
& df & 253 \\
& Sig. &, 000 \\
\hline
\end{tabular}

Fonte: elaborado pelos autores. 
Representando 70,9\% do total das variâncias das variáveis observáveis, a estrutura latente associada ao meio ambiente, constante da primeira seção do questionário, identificou três dimensões. Em função do que as variáveis alocadas a cada dimensão guardavam em comum, esses fatores foram definidos como Emoção, Social e Mobilidade.

O quadro 2 fornece a especificação dos construtos definidos como os fatores latentes exógenos, associados ao meio ambiente, a serem considerados no modelo a ser proposto posteriormente, bem como seus respectivos indicadores e o coeficiente alfa associado.

Quadro 2

Fatores latentes exógenos, seus indicadores e coeficientes alfa

\begin{tabular}{|l|l|c|}
\hline FATORES & INDICADORES & ALFA \\
\hline Emoção & $\begin{array}{l}\text { tolerante-exigente } \\
\text { acolhedor-hostil } \\
\text { tranquilo-estressante } \\
\text { satisfatório-irritante }\end{array}$ & 0,784 \\
\hline Social & $\begin{array}{l}\text { confiável-não confiável } \\
\text { recompensante-explorador } \\
\text { gentil-brutal }\end{array}$ & 0,806 \\
\hline Mobilidade & $\begin{array}{l}\text { ativo-passivo } \\
\text { estimulante-entediante } \\
\text { dinâmico-monótono }\end{array}$ & 0,730 \\
\hline
\end{tabular}

Fonte: elaborado pelos autores.

A operacionalização dos fatores latentes endógenos, relacionados ao estresse, foi realizada a partir dos sintomas apresentados na segunda seção do questionário. A análise fatorial, também exploratória, discriminou dois fatores explicando $37,5 \%$ do total das variâncias das variáveis observáveis. Pôde-se constatar que, de fato, o estresse poderia ser caracterizado a partir de duas fontes: o psicológico e o fisiológico. Os sintomas foram alocados ao fator (estresse) em função da carga fatorial prevalente. Nota-se que os sintomas relacionados ao sono ficaram todos associados ao fator psicológico de acordo com o método utilizado. O quadro 3 apresenta, como o anterior, os fatores, seus indicadores e o coeficiente alfa associado. 


$$
\text { Quadro } 3
$$

Fatores latentes endógenos, seus indicadores e coeficientes alfa

\begin{tabular}{|c|c|c|}
\hline FATOR (ESTRESSE) & INDICADOR (SINTOMA) & ALFA \\
\hline Psicológico & $\begin{array}{l}\text { sonolência } \\
\text { acorda cedo demais } \\
\text { dificuldade para dormir } \\
\text { dificuldade em manter a atenção } \\
\text { fastio }\end{array}$ & 0,624 \\
\hline Fisiológico & $\begin{array}{l}\text { dor de barriga } \\
\text { tonteira } \\
\text { enjoo } \\
\text { dor nas costas } \\
\text { falta de ar } \\
\text { queimação no estômago } \\
\text { coceira no corpo } \\
\text { arrepios }\end{array}$ & 0,696 \\
\hline
\end{tabular}

Fonte: elaborado pelos autores.

\section{O modelo}

A identificação dos estressores e dos sintomas do estresse, bem como a forma como eles se relacionam, foi modelada de acordo com o modelo de equações estruturais (SEM). A utilização do SEM em pesquisas sobre estresse ocupacional não é frequente. A maioria dos estudos nesta área utiliza métodos mais simplificados, como análise fatorial exploratória e, na maior parte dos casos, distribuição de frequências. No entanto, a literatura aponta uma preocupação expressa pela necessidade de utilização de métodos mais confiáveis nas pesquisas sobre estresse ocupacional (Bunce e Stephenson, 2000; Evers, Frese e Cooper, 2000).

Identificados os fatores ambientais que supostamente poderiam influenciar a ocorrência de estresse, partiu-se para se testar as hipóteses sobre as possíveis influências de cada um deles. As hipóteses a serem testadas foram definidas como: 
$\mathrm{H}_{\mathrm{o1}}$ : O fator emocional, verificado nos ambientes de trabalho, tem efeito sobre o estresse ocupacional;

$\mathrm{H}_{\mathrm{o} 2}: \mathrm{O}$ fator social, verificado nos ambientes de trabalho, tem efeito sobre o estresse ocupacional; e

$\mathrm{H}_{\mathrm{o}}$ : O fator mobilidade, verificado nos ambientes de trabalho, tem efeito sobre o estresse ocupacional.

Entende-se como estresse, para o desenvolvimento dos testes, a ocorrência de um conjunto de sintomas presentes no cotidiano dos servidores. Para as três afirmativas acima foram consideradas as duas formas de estresse, psicológico e fisiológico, tais como identificadas nas análises preliminares, por meio da avaliação exploratória.

Para se testar as hipóteses acima, foi elaborado um modelo de equações estruturais (SEM), no qual cada variável latente exógena, expressa pelos fatores ambientais, representa uma possível causa para cada uma das variáveis latentes endógenas, expressas pelas duas formas de estresse. O método de análise proposto tem se propagado sobremaneira nos últimos 10 anos nas diversas áreas da administração. Os estudos das relações simultâneas envolvendo variáveis observáveis e latentes receberam um grande impulso, principalmente após os trabalhos de Wright (1960), Jöreskog (1973), Keesing (1972) e Wiley (1973), que levaram ao desenvolvimento do aplicativo Lisrel, por Jöreskog e Sörbom (1996), precursor dos pacotes estatísticos que o sucederam, tais como Amos, EQS, Liscomp e outros.

Este modelo, em sua forma geral, é constituído por dois submodelos: um de medidas e outro estrutural. O primeiro permite medir as variáveis latentes a partir de seus indicadores, com base nos instrumentos da Análise Fatorial Confirmatória, enquanto o segundo utiliza tais mensurações para avaliar as possíveis relações entre as variáveis latentes. A especificação do modelo é a parte fundamental do processo, pois a partir dela é que as relações a serem testadas são efetivamente postuladas. Outro fator importante é a avaliação da capacidade de o modelo ter seus parâmetros estimados (identificados) com as informações fornecidas pelas associações entre as variáveis observáveis. A dinâmica das relações entre as variáveis observáveis é que permitirá, em última forma, avaliar a significância das relações estruturais que se deseja testar.

Para o presente estudo, o modelo especificado foi definido de acordo com o diagrama exposto na figura 1. 


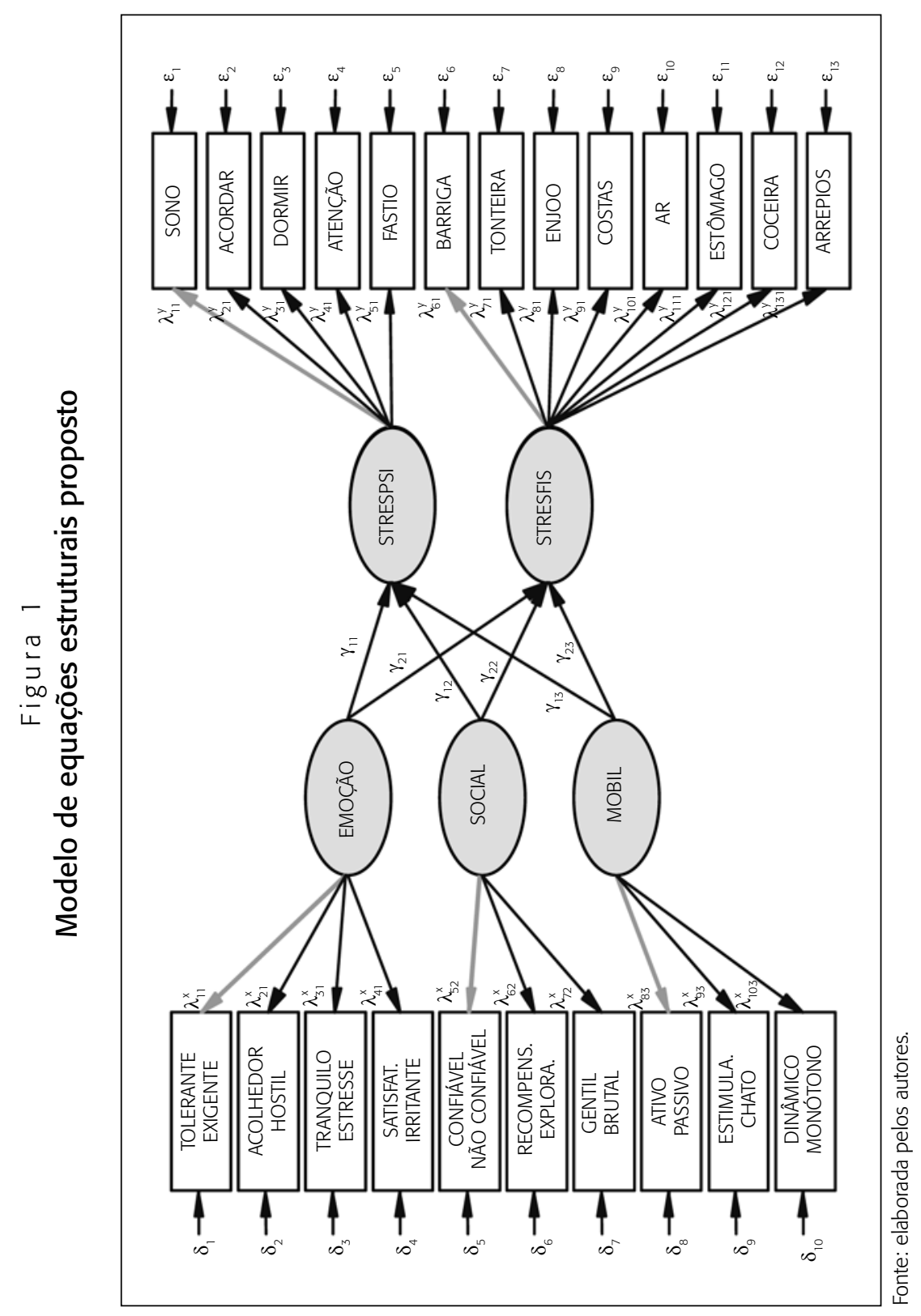


De acordo com o diagrama representativo do modelo, pela parte estrutural, os fatores ambientais, representados pelas dimensões emoção, social e mobilidade, têm efeito sobre as duas formas de estresse. A parte confirmatória do modelo foi construída com base nos resultados da análise exploratória que extraiu os fatores a partir dos indicadores, tanto para as variáveis exógenas, ambientais, quanto para as endógenas, relacionadas aos sintomas.

\section{A análise dos dados}

De acordo com Jöreskog e Sörbom (1996), a medida de associação indicada para variáveis com nível ordinal de mensuração, como as utilizadas no presente estudo, é a correlação policórica. A matriz das correlações utilizada como input do modelo encontra-se disposta no quadro 4.

Tendo como base o modelo apresentado na figura 1, foi utilizado o programa Lisrel para se estimar os coeficientes associados às relações estabelecidas. Primeiramente foram obtidos os coeficientes dos submodelos de medidas, para as três variáveis latentes exógenas, emoção, social e mobilidade, e para as variáveis latentes endógenas, estresse psicológico e estresse fisiológico. Todos foram significativos a $5 \%$.

$\mathrm{O}$ quadro 5 apresenta o resumo dos valores dos coeficientes (cargas fatoriais), os erros padronizados, os valores da estatística t a eles associados e os coeficientes padronizados (cargas padronizadas) para as variáveis latentes exógenas. Para se fixar a escala das variáveis latentes, foi atribuído valor unitário para as relações entre as primeiras variáveis indicadoras em relação aos conceitos por elas mensurados. Esta é também uma condição suficiente para a identificação dos coeficientes, segundo Bollen (1989).

De acordo com os resultados do quadro 5 , notamos que todos os indicadores carregaram os respectivos fatores latentes de modo significativo a 5\%. Para permitir uma avaliação comparativa entre os indicadores com maiores impactos para cada fator, foram calculados os coeficientes padronizados. Nota-se que, para o fator emoção, o indicador tranquilo-estressante desempenhou um papel mais importante do que os demais, com carga padronizada de 0,82. Gentil-brutal foi o fator mais influente para o fator social $(0,93)$ e dinâmico-monótono para o fator mobilidade, com carga padronizada de 0,73 . 
$\stackrel{M}{\sim}$

ส

$\bar{N}$

ำ

a

$\stackrel{\infty}{\infty}$

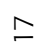

$\underline{\varphi}$

$\stackrel{n}{\underline{n}}$

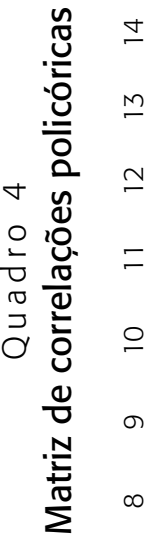

$\wedge$

6

เก

$\checkmark$

+

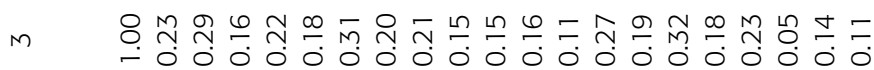

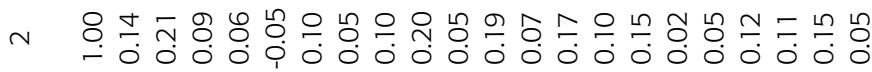
훔

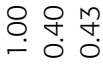

ํ.-

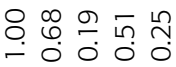

8 \&

৪

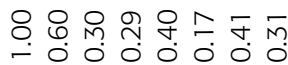

৪ \&

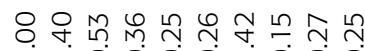

\&

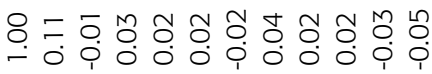

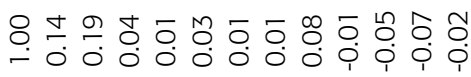

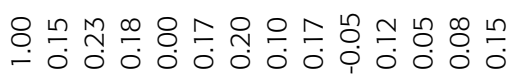

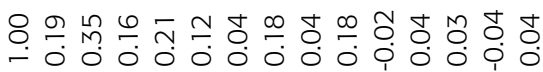

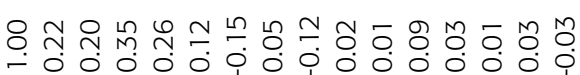

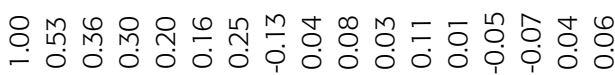

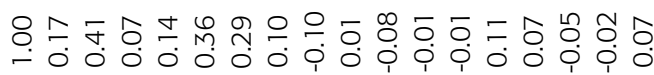

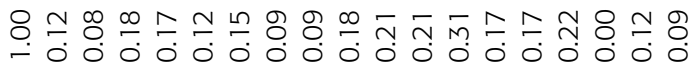

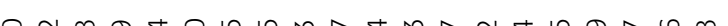

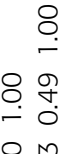
N

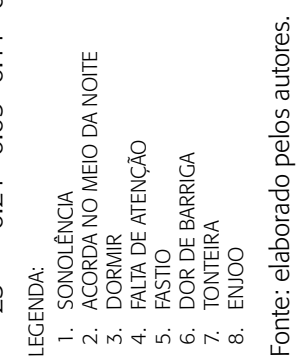




\section{Quadro 5}

\section{Coeficientes, erros e estatísticas t das variáveis latentes exógenas}

\begin{tabular}{|llcccc|}
\hline FATORES & \multicolumn{1}{c}{$\begin{array}{c}\text { INDICADORES } \\
\text { (AMBIENTAIS) }\end{array}$} & $\begin{array}{c}\text { CARGA } \\
\text { FATORIAL }\end{array}$ & $\begin{array}{c}\text { ERRO } \\
\text { PADRÃO }\end{array}$ & $\begin{array}{c}\text { CALOR t } \\
\text { Emoção }\end{array}$ & $\begin{array}{c}\text { CARGA } \\
\text { PADRÃO }\end{array}$ \\
& tolerante-exigente & 1,00 & - & - & 0,61 \\
& acolhedor-hostil & 1,04 & 0,14 & 7,53 & 0,63 \\
& tranquilo-estressante & 1,36 & 0,16 & 8,54 & 0,82 \\
& satisfatório-irritante & 1,17 & 0,14 & 8,14 & 0,71 \\
\hline Social & confiável- & 1,00 & - & - & 0,68 \\
& não confiável & 1,07 & 0,11 & 10,09 & 0,73 \\
& recompensante-explorador & 1,38 & 0,14 & 9,88 & 0,93 \\
& gentil-brutal & 1,00 & & & 0,59 \\
Mobilidade & ativo-passivo & 1,15 & 0,18 & 6,47 & 0,68 \\
& estimulante-entediante & 1,24 & 0,20 & 6,32 & 0,73 \\
\hline
\end{tabular}

Fonte: elaborado pelos autores.

Para as variáveis latentes endógenas, foram obtidos os valores descritos no quadro 6. Como no caso anterior, todos os indicadores carregaram significativamente a $5 \%$ nos dois fatores. Sonolência foi o sintoma com maior impacto sobre o estresse psicológico, com carga padrão de 0,64, seguido de falta de atenção com 0,60. Para o estresse fisiológico o sintoma mais influente foi enjoo, com carga padrão igual a 0,70, seguido de tonteira com 0,61.

Quadro 6

Coeficientes, erros e estatísticas t das variáveis latentes endógenas

\begin{tabular}{|llcccc|}
\hline $\begin{array}{l}\text { FATORES } \\
\text { (ESTRESSE) }\end{array}$ & \multicolumn{1}{c}{$\begin{array}{c}\text { INDICADORES } \\
\text { (SINTOMA) }\end{array}$} & $\begin{array}{c}\text { CARGA } \\
\text { FATORIAL }\end{array}$ & $\begin{array}{c}\text { ERRO } \\
\text { PADRÃO }\end{array}$ & $\begin{array}{c}\text { VALOR } \\
\mathrm{t}\end{array}$ & $\begin{array}{c}\text { CARGA } \\
\text { PADRÃO }\end{array}$ \\
\hline Psicológico & sonolência & 1,00 & -- & -- & 0,64 \\
& acorda no meio da noite & 0,43 & 0,12 & 3,45 & 0,27 \\
& dormir & 0,77 & 0,13 & 5,76 & 0,49 \\
& falta de atenção & 0,94 & 0,14 & 6,57 & 0,60 \\
& fastio & 0,82 & 0,14 & 5,99 & 0,52 \\
\hline Fisiológico & dor de barriga & 1,00 & -- & -- & 0,47 \\
& tonteira & 1,30 & 0,23 & 5,74 & 0,61 \\
& enjoo & 1,49 & 0,25 & 6,03 & 0,70 \\
& dor nas costas & 1,01 & 0,20 & 5,02 & 0,48 \\
& falta de ar & 0,75 & 0,18 & 4,11 & 0,35 \\
& dor de estômago & 1,11 & 0,21 & 5,29 & 0,52 \\
& coceira & 0,78 & 0,18 & 4,24 & 0,37 \\
& arrepios & 0,65 & 0,18 & 3,69 & 0,31 \\
\hline
\end{tabular}

Fonte: elaborado pelos autores. 
A significância dos coeficientes do modelo estrutural é que permitirá testar as hipóteses levantadas quanto aos efetivos efeitos dos estresses psicológico e fisiológico. Abaixo são apresentados os coeficientes estimados para as relações estruturais na forma definida pelo modelo.

Quadro 7

Coeficientes estimados para as relações estruturais

\begin{tabular}{|lcccc|}
\hline ESTRESSE & $\begin{array}{c}\text { FATORES } \\
\text { AMBIENTAIS }\end{array}$ & COEFICIENTES & $\begin{array}{c}\text { ERRO } \\
\text { PADRÃO }\end{array}$ & ESTATÍSTICA t \\
\hline Psicológico & emoção & 0,35 & 0,10 & 3,55 \\
& social & 0,15 & 0,08 & 1,93 \\
& mobilidade & 0,03 & 0,10 & 0,33 \\
\hline \multirow{2}{*}{ Fisiológico } & emoção & 0,03 & 0,06 & 0,47 \\
& social & 0,03 & 0,05 & 0,60 \\
& mobilidade & $-0,02$ & 0,07 & $-0,25$ \\
\hline
\end{tabular}

Fonte: elaborado pelos autores.

Do quadro 7, nota-se que os fatores ambientais não se mostraram significativos para explicar o estresse fisiológico, a 5\%. Ademais, somente a dimensão emoção mostrou ter efeito sobre o estresse psicológico, embora o fator social tenha ficado no limite da região de rejeição. Pesquisas futuras podem melhor avaliar a significância deste fator como possível fonte de estresse psicológico. De acordo com os resultados obtidos a partir do modelo estrutural, pode-se constatar que apenas a hipótese 1 , para o estresse psicológico, pôde ser confirmada, ou seja, o fator ambiental relativo à emoção é o único que, efetivamente, leva ao estresse psicológico, não causando, porém, o estresse fisiológico. A figura 2 resume o modelo proposto, representando seus coeficientes e erros. 


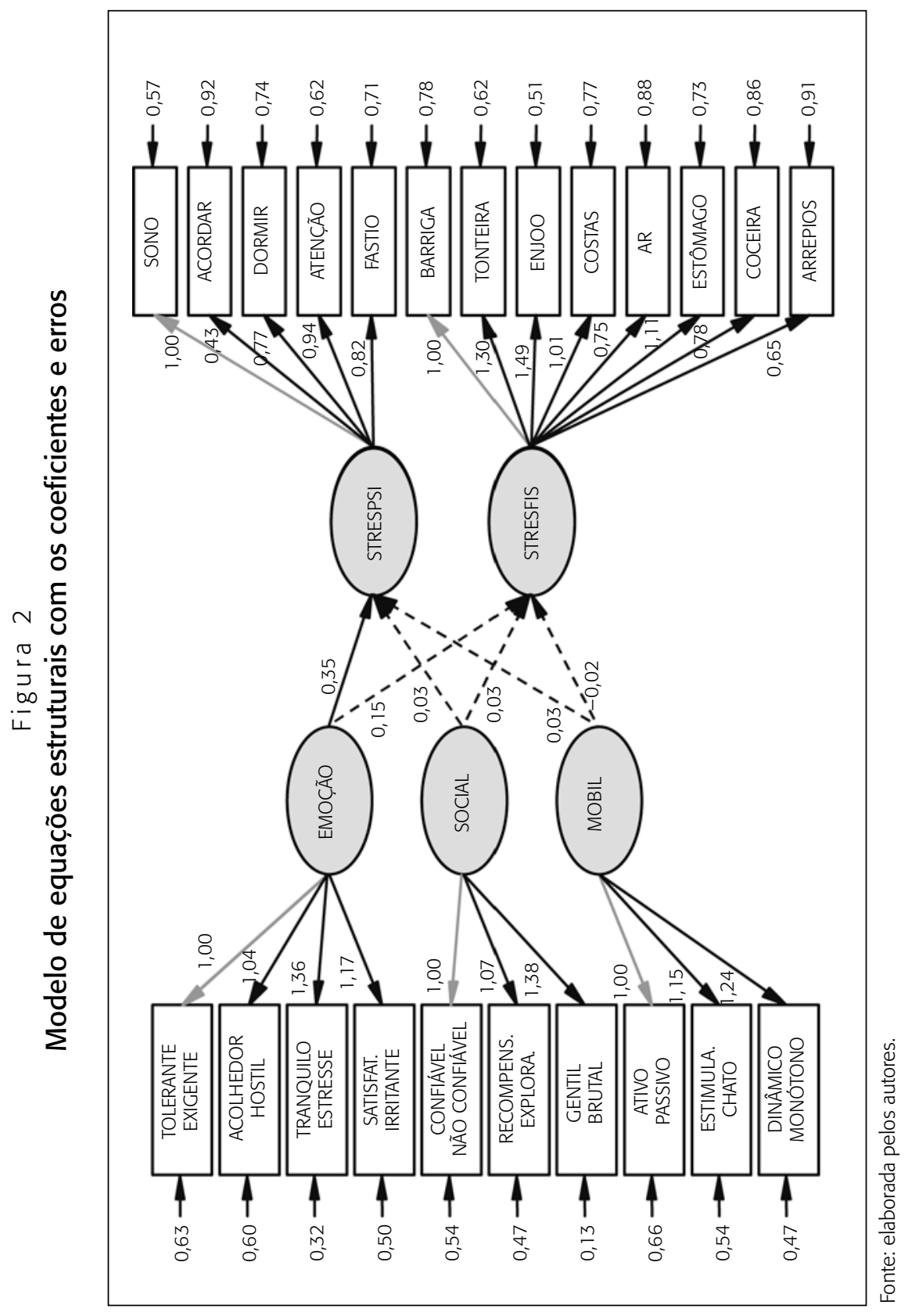




\section{Considerações finais}

Algumas observações sobre a questão do estresse ocupacional no âmbito da administração pública brasileira podem ser feitas. Em síntese, a pesquisa mostra que apenas o fator emocional presente no ambiente de trabalho nas organizações públicas influencia o estresse ocupacional psicológico, entre os três fatores estudados. $\mathrm{O}$ fator social merece atenção em investigações futuras sobre o tema, dado o fato de sua significância ter ficado na fronteira da rejeição.

Em seus contornos mais amplos, a pesquisa identificou as relações entre três fatores ambientais (emocional, social e mobilidade) e duas formas de estresse (psicológico e fisiológico), operacionalizadas pelos indicadores do ambiente organizacional e pelos sintomas de estresse, respectivamente.

Os resultados obtidos encontram respaldo na literatura especializada no tema, ainda que não sigam na íntegra qualquer modelo prevalente na análise do estresse ocupacional. A opção pela proposta de uma nova perspectiva de análise deve-se ao fato de se ter privilegiado a identificação dos estressores baseados no ambiente de trabalho, em face do interesse específico na área de administração.

Foram confirmados como estressores organizacionais fatores intrínsecos ao trabalho e relacionados ao papel do indivíduo na organização, conforme Cooper e Marshall (1976) já haviam identificado.

A iniciativa do estudo permite que se tenha uma diretriz preliminar para ações gerenciais voltadas à minimização das consequências do estresse ocupacional no setor público, tais como: (i) o aumento dos custos previdenciários advindo do absenteísmo e dos afastamentos por problemas de saúde; e (ii) a redução do desempenho e da qualidade de trabalho do servidor.

A pesquisa, desenvolvida por um método estatístico que privilegia a confiabilidade do modelo, ainda pode ser aprimorada no que diz respeito aos sintomas. Embora os sintomas tenham mostrado duas dimensões distintas, identificadas como psicológica e fisiológica, esta última não foi explicada por qualquer dos fatores ambientais considerados. Talvez outras variáveis não associadas ao estresse concorram para estes sintomas fisiológicos, ou até mesmo estes possam ser caracterizados simplesmente como uma somatização daqueles, o que pode ser avaliado em estudos futuros. Outra possibilidade pode ser atribuída ao fato de que, embora o indivíduo perceba o ambiente de forma supostamente estressante, o estresse de fato não ocorre. Isto pode acontecer se o indivíduo não mais reagir negativamente aos estressores. Outros possíveis sintomas podem ter sido omitidos, razão pela qual a variação total dos indica- 
dores de estresse explicada pelos dois fatores, estresse psicológico e estresse fisiológico, foi de apenas 37,5\%.

Por fim, pesquisas futuras poderão incorporar variáveis psicológicas, relacionadas à personalidade do indivíduo, que potencializem o estresse neste modelo. A identificação de fatores externos que possam influenciar os sintomas de estresse fisiológico seria, da mesma forma, de grande contribuição para um melhor mapeamento de um problema que aflige grande parte dos servidores públicos, em todos os níveis de governo.

\section{Referências}

ADMI, H.; MOSHE-EILON, Y. Stress among charge nurses: tool development and stress measurement. Nursing Economic\$, v. 28, n. 3, May/June 2010.

ALBRECHT, K. A. O gerente e o estresse: faça o estresse trabalhar para você. Rio de Janeiro: Jorge Zahar, 1990.

ARNOLD, J.; COOPER, C.L.; ROBERTSON, I.T. Work psychology: understanding human behavior in the workplace. 2nd. ed. London: Pitman, 1995.

AYRES, K.V. et al. Stress ocupacional no ambiente acadêmico universitário: um estudo em professores universitários com cargo de chefia intermediária. In: ENCONTRO ANUAL DA ASSOCIAÇÃO NACIONAL DOS PROGRAMAS DE PÓS-GRADUAÇÃO EM ADMINISTRAÇÃO, 1999. Anais... Anpad, 1999.

BOLLEN, D.A. Structural equations with latent variables. New York: John Wiley e Sons, Inc., 1989.

BRASIL. MINISTÉRIO DO PLANEJAMENTO, ORÇAMENTO E GESTÃO. Boletim Estatístico de Pessoal, n. 173, set. 2010. Disponível em: <www.servidor.gov.br/publicacao/boletim_estatistico/bol_estatistico_10/Bol173_Set2010.pdf.>.

BUNCE, D.; STEPHENSON, K. Statistical considerations in the interpretation of research on occupational stress management interventions. Work \& Stress, v. 14, n. 3, p. 197-212, 2000.

CAMPBELL, D.J.; NOBEL, O.B. Occupational stressors in military service: a review and framework. Military Psychology, v. 21, Suppl. 2, p. S47-S67, 2009.

CAVANAUGH, M.A. et al. An empirical examination of self-reported work stress among U.S. managers. Journal of Applied Psychology, n. 85, p. 65-74, 2000.

CHEN, C.K. et al. A study of job stress coping strategies, and job satisfaction for nurses working in middle-level hospital operating rooms. Journal of Nursing Research, v. 17, n. 3, 2009. 
COHEN, S. et al. Measuring stress. Oxford: Oxford University Press, 1995.

COOPER, C.L.; BAGLIONI, A.J. A structural model approach towards the development of a theory of the link between stress and mental health. British Journal of Medical Psychology, v. 61, n. 2, p. 87-102, 1988.

et al. Living with stress. London: Peguin Books, 1988.

et al. Occupational stress, job satisfaction and well-being in anaesthetists.

Stress Medicine, v. 15, p. 115-126, 1999.

et al. Organizational stress: a review and critique of theory, research and applications. London: Sage, 2001.

; MARSHALL, J. Occupational sources of stress: a review of the literature relating to coronary heart disease and mental ill health. Journal of Occupational Psychology, v. 49, p. 11-28, 1976.

COSTA, M. et al. Estresse: diagnóstico dos policiais militares em uma cidade brasileira. Revista Panamericana de Saúde Pública, v. 21, n. 4, p. 217-222, 2007.

COUTO, H.A.; MORAES, F.R. Stress no trabalho, fatores psicossociais e alta incidência de LER/Dort entre operadoras de caixa de supermercado: um estudo de caso. In: ENCONTRO ANUAL DA ASSOCIAÇÃO NACIONAL DOS PROGRAMAS DE PÓS-GRADUAÇÃO EM ADMINISTRAÇÃO, 1999. Anais... Anpad, 1999.

COUTO, J.H.; PAIVA, K.C.M. Qualidade de vida e estresse gerencial "pós-choque de gestão": o caso da Copasa-MG. Revista de Administração Pública, v. 42, n. 6, p. 1189-1211, nov./dez. 2008.

CRONBACH, L.J. Coefficient alpha and the internal structure of test. Phychometrika, n. 12, p. 1-16, 1951.

DEJOURS, C. A loucura do trabalho: estudo de psicopatologia do trabalho. 5. ed. São Paulo: Cortez-Oboré, 1992.

. Uma nova visão do sofrimento humano nas organizações. In: CHANLAT, J.F. (Coord.). O indivíduo na organização: dimensões esquecidas. 3. ed. São Paulo: Atlas, 1996. v. 1, p. 149-173.

ELKINS, G. et al. Perceived stress among nursing and administration staff related to accreditation. Clin Nurs Res, July 2010.

EVANS, O.; STEPTOE, A. Social support at work, heart rate, and cortisol: a self-monitoring study. Journal of Occupational Health Psychology, v. 6, p. 361-370, 2001.

EVERS, A.; FRESE, M.; COOPER, C.L. Revisions and further developments of the occupational stress indicator: Lisrel results from four dutch studies. Journal of Occupational and Organizational Psycology, n. 73, p. 221-240, 2000. 
FIGUEROA, N.L. et al. Um instrumento para a avaliação de estressores psicossociais no contexto de emprego. Psicologia: Reflexão e Crítica, v. 14, n. 3, p. 653-659, 2001.

FRIED, Y. et al. The interactive effect of role conflict and role ambiguity on job performance. Journal of Occupational and Organizational Psychology, n. 71, p. 1927, 1998.

FULCHERI, M. et al. Stress and managerial work: organizational culture and technological changes: a clinical study. Journal of Managerial Psychology, v. 10, n. 4, p. 3-8, 1995.

GLOWINKOWSKI, S.P.; COOPER, C.L. Managers and professionals in business/industrial settings: the research evidence. In: IVANCEVICH J.M.; GANGSTER, D.C. (Org.). Job stress: from theory to suggestion. Nova York: Haworth, 1987.

IWATA, N.; SUZUKI, K. Role stress - mental health relations in Japanese bank workers: a moderating effect of social support. Applied Psychology: An International Review, n. 45, p. 207-218, 1997.

JEX, S.M. Stress and job performance. London: Sage, 1998.

JOHNSON, S. et al. The experience of work-related stress across occupations. Journal of Managerial Psychology, v. 20, n. 2, p. 178-187, 2005.

JÖRESKOG, K.G. A general method for estimating a linear structural equation system. In: GOLDBERG, A.S.; DUNCAM, O.D. (Ed.). Structural equation models in the social sciences. New York: Academic Press, 1973. p. 105-112.

JÖRESKOG, K.; SÖRBOM, D. Lisrel 8: user's reference guide. Scientific Software International, Il, 1996.

KAHN, W.A. Caring for the caregivers: patterns of organizational caregiving. Administrative Science Quarterly, v. 38, n. 4, p. 539-564, 1993.

KEESING, J.W. Maximum likelihood approaches to causal analysis. Ph.D. dissertation — Department of Education, University of Chicago, 1972.

LARANJEIRA, C.A. An integrated perspective of the organisational context and experience of stress. Revista Salud Pública, v. 11, n. 1, p. 123-133, 2009.

LIPP, M.E.; NOVAES, T.; SACRAMENTO, M. Stress e qualidade de vida em magistrados da justiça do trabalho: diferenças entre homens e mulheres. Psicologia: Reflexão e Crítica, v. 15, n. 3, p. 537-548, 2002.

; TANGANELLI, M.S. Stress e qualidade de vida em magistrados da justiça do trabalho: diferenças entre homens e mulheres. Psicologia: Reflexão e Crítica, v. 15, n. 3, p. 537-548. 2002. 
LOUGHBOROUGH UNIVERSITY. Occupational stress policy. 2009. Disponível em: <www.lboro.ac.uk/admin/personnel/stress/stresspolicy.html>.

MACKIE, K.S.; HOLAHAN, C.K.; GOTTLIEB, N.H. Employee involvement management practices, work stress, and depression in employees of a human service residential care facility. Human Relations, n. 54, p. 1065-1092, 2001.

MARQUES, A.L.; PAIVA, K.C.M. Público x privado: qualidade de vida, stress e situação de trabalho de professores de instituição de ensino superior. In: ENCONTRO ANUAL DA ASSOCIAÇÃO NACIONAL DOS PROGRAMAS DE PÓS-GRADUAÇÃO EM ADMINISTRAÇÃO, 1999. Anais... Anpad, 1999.

MENG-JINN, C.; CUNRADI, C. Job stress, burnout and substance use among urban transit operators: the potential mediating role of coping behavior. Work \& Stress, v. 22, n. 4, 2008.

MORAES, L.F.R.; KILIMNIK, Z.M. As dimensões básicas do trabalho, qualidade de vida e stress: uma análise em profissões de impacto na sociedade. Belo Horizonte: Cepead/UFMG, 1992. (Relatório de pesquisa)

MOTA, C.M. et al. Estresse e sofrimento no trabalho dos executivos. Psicologia em Revista, v. 14, n. 1, 2008.

NEW YORK STATE PUBLIC EMPLOYEES FEDERATION. Occupational stress factsheet. 2010. Disponível em: <www.nyspef.org/healthandsafety/files/stress_factsheet.pdf $>$.

PASCHOAL, T.; TAMAYO, A. Validação da escala de estresse no trabalho. Estudos de Psicologia, v. 9, n. 1, jan./abr. 2004.

POLLARD, T.M. Changes in mental well-being, blood pressure and total cholesterol levels during workplace reorganization: The impact of uncertainty. Work \& Stress, v. 15, p. 14-28, 2001.

QUICK, J. et al. O executivo em harmonia. São Paulo: Publifolha, 2003.

RAMIREZ, M.T.G. et al. Modelo estructural predictor de la salud mental y física en mujeres. Revista Panamericana de Saúde Pública, v. 23, n. 2, p. 101-108, 2008.

; HERNANDEZ, R.L. Confirmación de un modelo explicativo del estrés y de los síntomas psicosomáticos mediante ecuaciones estructurales. Revista Panamericana de Saúde Pública, v. 23, n. 1, p. 7-18, 2008.

REES, D.W. Work-related stress in health service employees. Journal of Managerial Psychology, v. 10, n. 3, p. 4-11, 1995.

REES, C.J.; REDFERN, D. Recognising the perceived causes of stress - a training and development perspective. Industrial and Commercial Training, v. 32, n. 4, p. 120-127, 2000. 
SANTOS, C.M.Q. et al. Qualidade de vida no trabalho, estresse ocupacional e o sistema just in time: um estudo de caso no setor automobilístico mineiro. In: ENCONTRO ANUAL DA ASSOCIAÇÃO NACIONAL DOS PROGRAMAS DE PÓSGRADUAÇÃO EM ADMINISTRAÇÃO, 1999. Anais... Anpad, 1999.

SEEGERS, G.; VAN ELDEREN, T. Examining a model of stress reactions of bank directors. European Journal of Psychological Assessment, v. 12, n. 3, p. 212-223, 1996.

SKAKON, J. et al. Do managers experience more stress than employees? Results from the Intervention Project on Absence and Well-being (IPAW) study among Danish managers and their employees. Work: A Journal of Prevention, Assessment and Rehabilitation, v. 38, n. 2, p. 103-109, 2011.

SPIELBERGER, C.; REHEISER, E.C. The job stress survey. Measuring gender differences in occupation stress. Journal of Social Behavior and Personality, v. 9, p. 199-218, 1994.

STEINMETZ, H.; SCHMIDT, P. Subjective health and its relationship with working time variables and job stressors: Sequence or general factor model? Work \& Stress, v. 24, n. 2, 2010.

THEORELL, T. Psychosocial and physiological factors in relation to blood pressure at rest. A study of swedish men in the twenties. Journal of Hypertension, v. 3, p. 591-600, 1985.

TRAVERS, C.J.; COOPER, C.L. Mental health, job satisfaction and occupational stress among UK teachers. Work \& Stress, v. 7, n. 3, p. 203-219, 1993.

VELOSO, H.M.; PIMENTA, S.M. Fontes de pressão e as novas configurações do trabalho: uma discussão sobre os modelos de análise do estresse ocupacional frente a realidade bancária. In: Anais da Associação Nacional dos Programas de Pós-Graduação em Administração. Curitiba: 2004.

WANG, J.L.; PATTEN, S.B. Perceived work stress and major depression in the Canadian employed population, 20-49 years old. Journal of Occupational Health Psychology, v. 6, p. 283-289, 2001.

WILEY, D.E. The identification problem for structural equation models with unmeasured variables. In: GOLDBERGER, A.S.; DUNCAM, O.D. (Ed.). Structural Equation Models in the Social Sciences. New York: Academic Press, 1973. p. 69-83.

WRIGHT, S. Path coefficients and path regressions: Alternative or complementary concepts? Biometrics, n. 16, p. 189-202, 1960. 